\title{
Metric of a Slow Rotating Body with Quadrupole Moment from the Erez-Rosen Metric
}

\author{
Francisco Frutos-Alfaro", Edwin Retana-Montenegro, Iván Cordero-García, Javier Bonatti-González \\ School of Physics, University of Costa Rica, San Pedro, Costa Rica \\ Email: frutos@fisica.ucr.ac.cr
}

Received September 2, 2013; revised October 1, 2013; accepted October 10, 2013

Copyright (C) 2013 Francisco Frutos-Alfaro et al. This is an open access article distributed under the Creative Commons Attribution License, which permits unrestricted use, distribution, and reproduction in any medium, provided the original work is properly cited.

\begin{abstract}
A metric representing a slowly rotating object with quadrupole moment is obtained using a perturbation method to include rotation into the weak limit of the Erez-Rosen metric. This metric is intended to tackle relativistic astrometry and gravitational lensing problems in which a quadrupole moment has to be taken into account.
\end{abstract}

Keywords: General Relativity; Einstein Field Equations; Quadrupole Moment; Erez-Rosen Metric

\section{Introduction}

The first quadrupole solution to the Einstein field equations (EFE) was found by Erez and Rosen [1]. Some errors were found in this derivation. These were later corrected by Doroshkevich et al. [2], Winicour et al. [3] and Young and Coulter [4]. Other multipole solutions to the EFE were obtained by Quevedo [5,6], Quevedo and Mashhoon [7], and Castejón-Amenedo et al. [8]. In the first three articles, the solutions were obtained with the help of the Hoenselaers-Kinnersley-Xanthopoulos (HKX) transformations [9], while in the latter, they used the Ernst formalism [10]. These authors obtain new metrics from a given seed metric. One can include other desirable characteristics (rotation, multipole moments, etc.) to these seed metrics by means of these formalisms. Recently, Boshkayev et al. [11] obtained an approximate solution describing the interior and exterior gravitational field of a slowly rotating and slightly deformed object.

The aim of this article is to derive an appropriate analytical tractable metric for calculations in astrometry and gravitational lens theory including the quadrupole moment and rotation in a natural form. For this new rotating metric, it is not necessary for a multipolar expansion in the potential to include the multipolar terms because the seed metric already has a quadrupole term, that is to say this metric is multipolar intrinsically.

This paper is organized as follows. In Section 2, we get the weak limit of the Erez-Rosen metric. The Lewis

*CINESPA (Space Research Center), University of Costa Rica.

${ }^{\#}$ CICANUM (Nuclear Research Center), University of Costa Rica. metric is presented in Section 3. The perturbation method is discussed in Section 4. The application of this method leads to a new solution to the EFE with quadrupole moment and rotation. It is checked by means of the REDUCE software [12] that the resulting metric is solution of the EFE. In Section 5, we compare our solution with the exterior Hartle-Thorne metric [13] in order to assure that our metric has astrophysical meaning. In Section 6 , we transform the obtained metric using Cartesian coordinates. Forthcoming works with this metric are discussed in Section 7.

\section{Weak Limit of the Erez-Rosen Metric}

The Erez-Rosen metric [3,4,14,15] represents a body with quadrupole moment. The principal axis of the quadrupole moment is chosen along the spin axis, so that gravitational radiation can be ignored. This metric is given by

$$
\begin{aligned}
\mathrm{d} s^{2}= & \mathrm{e}^{2 \psi} \mathrm{d} t^{2}-\mathrm{e}^{2(\gamma-\psi)} P\left(\frac{\mathrm{d} r^{2}}{r^{2}-2 M r}+\mathrm{d} \theta^{2}\right) \\
& -\mathrm{e}^{-2 \psi}\left(r^{2}-2 M r\right) \sin ^{2} \theta \mathrm{d} \varphi^{2},
\end{aligned}
$$

where $M$ is the mass of the object and

$$
\begin{gathered}
P=r^{2}-2 M r+M^{2} \sin ^{2} \theta \\
\psi=\frac{1}{2}\left(1+\frac{q}{4}\left(3 \lambda^{2}-1\right)\left(3 \mu^{2}-1\right)\right) \ln \left[\frac{\lambda-1}{\lambda+1}\right] \\
+\frac{3}{4} q \lambda\left(3 \mu^{2}-1\right),
\end{gathered}
$$




$$
\begin{aligned}
\gamma= & \frac{1}{2}(1+q)^{2} \ln \left[\frac{\lambda^{2}-1}{\lambda^{2}-\mu^{2}}\right]-\frac{3}{2} q\left(1-\mu^{2}\right)\left[\lambda \ln \left[\frac{\lambda-1}{\lambda+1}\right]+2\right] \\
& +\frac{9}{4} q^{2}\left(1-\mu^{2}\right)\left[\frac{1}{16}\left(\lambda^{2}-1\right)\left(\lambda^{2}+\mu^{2}-9 \lambda^{2} \mu^{2}-1\right)\right. \\
& \times \ln ^{2}\left[\frac{\lambda-1}{\lambda+1}\right]+\frac{\lambda}{4}\left(\lambda^{2}+7 \mu^{2}-9 \lambda^{2} \mu^{2}-\frac{5}{3}\right) \ln \left[\frac{\lambda-1}{\lambda+1}\right] \\
& \left.+\frac{\lambda^{2}}{4}\left(1-9 \mu^{2}\right)+\left(\mu^{2}-\frac{1}{3}\right)\right],
\end{aligned}
$$

with $\lambda=r / M-1$ and $\mu=\cos \theta$. From now on, we will keep in the derivations terms up to order $M^{2}$ and $q M^{3}$. The approximate forms of $\psi$ and $\gamma$ are

$$
\begin{aligned}
\psi= & \frac{1}{2} \ln \left(1-\frac{2 M}{r}\right)-\frac{2}{15} q \frac{M^{3}}{r^{3}} P_{2}(\cos \theta) \\
& +O\left(M^{3}, q M^{4}, q^{2}\right), \\
\gamma= & \frac{1}{2} \ln \left(\frac{r^{2}-2 M r}{\Delta}\right)+O\left(M^{3}, q M^{4}, q^{2}\right),
\end{aligned}
$$

where $q=15 G Q /\left(2 c^{2} M^{3}\right)$ with $Q$ representing the quadrupole moment, and $P_{2}(\cos \theta)=\left(3 \cos ^{2} \theta-1\right) / 2$ is the second Legendre polynomial.

We define the following variables

$$
\begin{aligned}
C= & \mathrm{e}^{-\chi} \simeq 1-\frac{2}{15} q \frac{M^{3}}{r^{3}} P_{2}(\cos \theta) \\
& +O\left(M^{3}, q M^{4}, q^{2}\right), \\
F= & \mathrm{e}^{2 \psi}=\left(1-\frac{2 M}{r}\right) \mathrm{e}^{-2 \chi} \\
\simeq & 1-\frac{2 M}{r}-\frac{4}{15} q \frac{M^{3}}{r^{3}} P_{2}(\cos \theta) \\
& +O\left(M^{3}, q M^{4}, q^{2}\right),
\end{aligned}
$$

and

$$
G=\mathrm{e}^{2 \gamma} \simeq \frac{r^{2}-2 M r}{\Delta}+O\left(M^{3}, q M^{4}, q^{2}\right),
$$

where

$$
\chi=\frac{2}{15} q \frac{M^{3}}{r^{3}} P_{2}(\cos \theta) .
$$

If we substitute the former definitions into (1), the metric takes the form

$$
\mathrm{d} s^{2}=F \mathrm{~d} t^{2}-\frac{\mathrm{d} r^{2}}{F}-r^{2} \mathrm{e}^{2 \chi} \mathrm{d} \Sigma^{2},
$$

where $\mathrm{d} \Sigma^{2}=\mathrm{d} \theta^{2}+\sin ^{2} \theta \mathrm{d} \varphi^{2}$ and the inverse of $F$ is written as

$$
\begin{aligned}
& \frac{1}{F}=\left(1-\frac{2 M}{r}\right)^{-1} \mathrm{e}^{2 \chi} \\
& \simeq 1+\frac{2 M}{r}+\frac{4 M^{2}}{r^{2}}+\frac{4}{15} q \frac{M^{3}}{r^{3}} P_{2}(\cos \theta) \\
& \quad+O\left(M^{3}, q M^{2}, q^{2}\right) .
\end{aligned}
$$

It is interesting to note that the spherical symmetry is not presented in the weak limit.

\section{The Lewis Metrics}

The Lewis metric is given by $[14,16]$

$$
\mathrm{d} s^{2}=V \mathrm{~d} t^{2}-2 W \mathrm{~d} t \mathrm{~d} \varphi-\mathrm{e}^{\mu} \mathrm{d} \rho^{2}-\mathrm{e}^{v} \mathrm{~d} z^{2}-Z \mathrm{~d} \varphi^{2},
$$

where we have chosen the canonical coordinates $x^{1}=\rho$ and $x^{2}=z, V, W, Z, \mu$ and $v$ are functions of $\rho$ and $z\left(\rho^{2}=\right.$ $V Z+W^{2}$ ). Choosing $\mu=v$ and performing the following changes of potentials.

$$
V=f, \quad W=\omega f, \quad Z=\frac{\rho^{2}}{f}-\omega^{2} f, \quad \mathrm{e}^{\mu}=\frac{\mathrm{e}^{\gamma}}{f},
$$

we get the Papapetrou metric

$$
\mathrm{d} s^{2}=f(\mathrm{~d} t-\omega \mathrm{d} \varphi)^{2}-\frac{\mathrm{e}^{\gamma}}{f}\left[\mathrm{~d} \rho^{2}+\mathrm{d} z^{2}\right]-\frac{\rho^{2}}{f} \mathrm{~d} \varphi^{2} .
$$

\section{Perturbing the Erez-Rosen Metric}

To include slow rotation into the Erez-Rosen metric we use the Lewis-Papapetrou metric (5). First of all, we choose expressions for the canonical coordinates $\rho$ and $z$. For the Kerr metric [17], one particular choice is $[6,18]$

$$
\rho=\sqrt{\Delta} \sin \theta, \quad z=(r-M) \cos \theta,
$$

where

$$
\begin{aligned}
& \Delta=r^{2}-2 M r+a^{2} \simeq r^{2}-2 M r=r^{2} F \mathrm{e}^{2 \chi} . \\
& \text { From (6) we get } \\
\mathrm{d} \rho^{2}+\mathrm{d} z^{2} & \\
= & {\left[(r-M)^{2} \sin ^{2} \theta+\Delta \cos ^{2} \theta\right] \times\left(\frac{\mathrm{d} r^{2}}{\Delta}+\mathrm{d} \theta^{2}\right) } \\
\simeq & \left(1+\frac{M^{2}}{r^{2}} \sin ^{2} \theta\right) \mathrm{d} r^{2}+r^{2}\left(1-\frac{2 M}{r}+\frac{M^{2}}{r^{2}} \sin ^{2} \theta\right) \mathrm{d} \theta^{2},
\end{aligned}
$$

where we have expanded up to $M^{2}$ order.

Now, let us choose $V=f=F$ and neglect the second order in $\omega\left(\omega^{2} \approx 0, W^{2} \approx 0\right)$. Then, we have

$$
Z \simeq \frac{\rho^{2}}{f}=r^{2} \mathrm{e}^{2 \chi} \sin ^{2} \theta
$$

If we choose

$$
\mathrm{e}^{\mu}=\frac{r^{2} \mathrm{e}^{2 \chi}}{(r-M)^{2} \sin ^{2} \theta+\Delta \cos ^{2} \theta},
$$


the term (7) becomes

$$
\mathrm{e}^{\mu}\left[\mathrm{d} \rho^{2}+\mathrm{d} z^{2}\right]=\frac{\mathrm{d} r^{2}}{F}+r^{2} \mathrm{e}^{2 \chi} \mathrm{d} \theta^{2} .
$$

This term appears in the approximate Erez-Rosen metric (3). From (5), let us propose the following metric

$$
\mathrm{d} s^{2}=V \mathrm{~d} t^{2}-2 W \mathrm{~d} t \mathrm{~d} \varphi-X \mathrm{~d} r^{2}-Y \mathrm{~d} \theta^{2}-Z \mathrm{~d} \varphi^{2},
$$

where $X=1 / V, Y=r^{2} \mathrm{e}^{2 \chi}$, and $Z=r^{2} \mathrm{e}^{2 \chi} \sin ^{2} \theta$.

We see that to obtain a slowly rotating version of the metric (3) the only potential we have to find is $W$. Then, the EFE must be solved:

$$
G_{i j}=R_{i j}-\frac{R}{2} g_{i j}=0,
$$

where $R_{i j}(i, j=0,1,2,3)$ are the Ricci tensor components and $R$ is the curvature scalar.

Fortunately, the Ricci tensor components $R_{00}, R_{11}, R_{12}$, $R_{22}, R_{33}$ and the curvature scalar $R_{s}$ depend upon the potentials $V, X, Y, Z$ and not on $W$. Therefore, these components vanish (see Appendix). The only remaining equation we have to solve is $R_{03}=0$, because it depends upon $W$. The equation for this component up to the order $O\left(M^{3}, a^{2}, q M^{4}, q^{2}\right)$ is

$$
\begin{aligned}
& 2\left(1-P_{2}(\cos \theta)\right)\left[\frac{\partial^{2} W}{\partial \theta^{2}}+r^{2} \frac{\partial^{2} W}{\partial r^{2}}\right] \\
& -3 \cos \theta \sin \theta \frac{\partial W}{\partial \theta}=0 .
\end{aligned}
$$

The solution for this equation is

$$
W=\frac{K}{r} \sin ^{2} \theta,
$$

where $K$ is a constant that we have to find. This constant can be found from the Lense-Thirring metric which can be obtained from the Kerr metric, i.e.

$$
\begin{aligned}
\mathrm{d} s^{2}= & \left(1-\frac{2 M}{r}\right) \mathrm{d} t^{2}+\frac{4 J}{r} \sin ^{2} \theta \mathrm{d} t \mathrm{~d} \varphi \\
& -\left(1-\frac{2 M}{r}\right)^{-1} \mathrm{~d} r^{2}-r^{2} \mathrm{~d} \Sigma^{2},
\end{aligned}
$$

where $J=M a$ is the angular momentum and $a$ is the rotation parameter.

Comparing the second term of the latter metric with the corresponding of the metric (8), i.e. $W$, we note that $K=-2 J=-2 M a$.

Then, the new rotating metric with quadrupole moment written in standard form [19] in spherical coordinates is

$$
\begin{aligned}
& \mathrm{d} s^{2}=\left(1-\frac{2 M}{r}\right) \mathrm{e}^{-2 \chi} \mathrm{d} t^{2}+\frac{4 J}{r} \sin ^{2} \theta \mathrm{d} t \mathrm{~d} \varphi \\
& -\left(1-\frac{2 M}{r}\right)^{-1} \mathrm{e}^{2 \chi} \mathrm{d} r^{2}-r^{2} \mathrm{e}^{2 \chi}\left(\mathrm{d} \theta^{2}+\sin ^{2} \theta \mathrm{d} \varphi^{2}\right) .
\end{aligned}
$$

We verified that the metric (10) is indeed a solution of the EFE using REDUCE [12] up to the order $O\left(a^{2}, q M^{4}\right.$, $q^{2}$ ). Hence, one does not need to expand the term $(1-2 M / r)^{-1}$ in a Taylor series.

\section{Comparison with the Exterior Hartle-Thorne Metric}

In order to establish whether our metric does really represent the gravitational field of an astrophysical object, one should show that it is possible to construct an interior solution, which can appropriately be matched with the exterior solution. For this purpose, we employ the exterior Hartle-Thorne metric $[13,20]$, which is given by

$$
\begin{aligned}
& \mathrm{d} s^{2}=\left(1-\frac{2 M_{0}}{r}+\frac{2 Q_{0} M_{0}^{3}}{r^{3}} P_{2}(\cos \theta)\right) \mathrm{d} t^{2} \\
& -\left(1+\frac{2 M_{0}}{r}+4 \frac{M_{0}^{2}}{r^{2}}-\frac{2 Q_{0} M_{0}^{3}}{r^{3}} P_{2}(\cos \theta)\right) \mathrm{d} r^{2} \\
& -r^{2}\left(1-\frac{2 Q_{0} M_{0}^{3}}{r^{3}} P_{2}(\cos \theta)\right) \mathrm{d} \Sigma^{2} \\
& +\frac{4 J_{0}}{r} \sin ^{2} \theta \mathrm{d} t \mathrm{~d} \varphi,
\end{aligned}
$$

where $M_{0}, J_{0}$, and $Q_{0}$ are related with the total mass, angular momentum, and mass quadrupole moment of the rotating object, respectively.

Now, comparing the exterior Hartle-Thorne metric with our expression (10), it can easily be seen that upon defining

$$
M_{0}=M, \quad J_{0}=J, \quad 2 Q_{0} M_{0}^{3}=-\frac{4}{15} q M^{3},
$$

both metrics coincide up to the order $O\left(M^{3}, a^{2}, q M^{4}, q^{2}\right)$. Our approximate expression for the Hartle-Thorne metric (11) was obtained by means of a REDUCE program using the expressions from Abramowicz et al. [21]. We compared these results with the approximate expression given by Boshkayev et al. [11] and found that they have an extra term of order $O\left(q M^{4}\right)$, which we neglected, because it is beyond the order we are working with. Additional differences are that our metric parameters $(M, J=$ $\left.M a, q M^{3}\right)$ are distinct and our expressions (12) are simpler than those of Boshkayev et al. [11].

\section{The Transformation of the Metric}

In some cases, the metric (10) has to be transform from spherical $(r, \theta, \varphi)$ into Cartesian coordinates $(x, y, z)$. For example, if a comparison with a post-Newtonian (PN) metric is made, we have to transform the metric (10) by using one of the following radial coordinates transformation: the harmonic or the isotropic coordinates of Schwarzschild metric. The first one is $r=R+M$, and the 
second one is $r=R(1+M / 2 R)^{2}$, where $R$ is a new radial coordinate [19]. We choose the first one, then the metric (10) is transformed into

$$
\begin{aligned}
& \mathrm{d} s^{2}=H \mathrm{~d} t^{2}+\frac{4 J}{R} \sin ^{2} \theta \mathrm{d} t \mathrm{~d} \varphi-\frac{\mathrm{d} R^{2}}{H} \\
& -R^{2} \mathrm{e}^{2 \chi}\left(1+\frac{M}{R}\right)^{2} \mathrm{~d} \Sigma^{2},
\end{aligned}
$$

where

$$
\begin{aligned}
H & =\left(\frac{1-\frac{M}{R}}{1+\frac{M}{R}}\right) \mathrm{e}^{-2 \chi} \\
\simeq & 1-\frac{2 M}{R}+\frac{2 M^{2}}{R^{2}}-\frac{4}{15} q \frac{M^{3}}{R^{3}} P_{2}(\cos \theta) \\
& +O\left(M^{3}, a^{2}, q M^{4}, q^{2}\right),
\end{aligned}
$$

and

$$
\begin{aligned}
& \frac{1}{H}=\left(\frac{1+\frac{M}{R}}{1-\frac{M}{R}}\right) \mathrm{e}^{2 \chi} \\
& \simeq 1+\frac{2 M}{R}+\frac{2 M^{2}}{R^{2}}+\frac{4}{15} q \frac{M^{3}}{R^{3}} P_{2}(\cos \theta) \\
& +O\left(M^{3}, a^{2}, q M^{4}, q^{2}\right) .
\end{aligned}
$$

Noting that $C=\exp (-\chi)$ is still given by (2) with $r$ changed by $R$. Now, transforming the metric (13) into the Cartesian coordinates which are given by the usual relations

$$
\begin{aligned}
& X=R \sin \theta \cos \varphi, \\
& Y=R \sin \theta \sin \varphi, \\
& Z=R \cos \theta .
\end{aligned}
$$

The resulting metric has the following form

$$
\begin{aligned}
& \mathrm{d} s^{2}=H \mathrm{~d} t^{2}+\frac{4 J}{R^{3}} \mathrm{~d} t(X \mathrm{~d} Y-Y \mathrm{~d} X) \\
& -\frac{P^{\prime}}{R^{2}}(\boldsymbol{X} \cdot \mathrm{d} \boldsymbol{X})^{2}-\left(1+\frac{M}{R}\right)^{2} \mathrm{e}^{2 \chi} \mathrm{d} \boldsymbol{X}^{2},
\end{aligned}
$$

where $\boldsymbol{X}=(X, Y, Z)$

$$
\begin{aligned}
& P^{\prime}=\frac{1}{H}-\left(1+\frac{M}{R}\right)^{2} \mathrm{e}^{2 \chi} \\
& \simeq \frac{M^{2}}{R^{2}}+O\left(M^{3}, a^{2}, q M^{4}, q^{2}\right) .
\end{aligned}
$$

The former metric can be generalized as follows

$$
\begin{aligned}
\mathrm{d} s^{2}= & H \mathrm{~d} t^{2}+8 \boldsymbol{V} \cdot \mathrm{d} \boldsymbol{X} \mathrm{d} t-\frac{P^{\prime}}{r^{2}}(\boldsymbol{X} \cdot \mathrm{d} \boldsymbol{X})^{2} \\
& -\left(1+\frac{M}{R}\right)^{2} \mathrm{e}^{2 \chi} \mathrm{d} \boldsymbol{X}^{2},
\end{aligned}
$$

where

$$
\begin{aligned}
& \left(1+\frac{M}{R}\right)^{2} \mathrm{e}^{2 \chi} \simeq 1+\frac{2 M}{R}+\frac{M^{2}}{R^{2}} \\
& +\frac{4}{15} q \frac{M^{3}}{R^{3}} P_{2}(\cos \theta)+O\left(M^{3}, a^{2}, q M^{4}, q^{2}\right),
\end{aligned}
$$

and the vector $\boldsymbol{V}$ is defined as

$$
\boldsymbol{V}=\frac{G}{2 c^{3} R^{3}}[\boldsymbol{J} \times \boldsymbol{X}],
$$

with $\boldsymbol{J}=J \boldsymbol{e}_{j}\left(\boldsymbol{e}_{j}\right.$ is an unit vector in the direction of $\left.\boldsymbol{J}\right)$.

\section{Summary and Conclusion}

In this paper, we include the rotational effect using the weak limit of the Erez-Rosen metric as seed metric into the Lewis-Papapetrou metric. Thus, a new metric with quadrupole moment and rotation in the weak limit is obtained. Generally speaking, the quadrupole moment is included in the metric, for instance, in gravitational lensing, through the expansion of the gravitational potential in a power series [22]. The resulting metric from our calculations includes the quadrupole moment in a natural form and is similar to the exterior metric obtained by Boshkayev et al. [11]. As we have seen in Section 4, our new metric agrees with the Hartle-Thorne solution [13], who obtained an interior metric that appropriately matches their exterior one, which guarantees the construction of an interior solution for our spacetime. This result indicates that our metric may be used to represent a compact astrophysical object. The new metric has many applications, for example, in calculations involving relativistic astrometry, in gravitational lens theory or planetary perihelion shift, and it is useful to have a metric that includes rotation and quadrupole moment. In relativistic astrometry, one needs a post-Newtonian metric to get some approximations from the deflection angle. It allows to get expressions for the right ascension $\alpha$ and declination $\delta$ for a celestial body $[23,24]$ in the gravitational field including rotation and quadrupole moment. In gravitational lens theory, the deflection angle [25,26] can be used to obtain the lens equation, thereby the lensing properties for this new metric with intrinsic gravitational quadrupole may be studied. Another application of this metric is to calculate the planetary perihelion shift [27]. These applications will be the aim of forthcoming works.

\section{REFERENCES}

[1] G. Erez and N. Rosen, "The Gravitational Field of a Particle Possessing a Multipole Moment," Bulletin of the Research Council of Israel, Vol. 8F, 1959, pp. 47-50.

[2] A. G. Doroshkevich, Ya. B. Zel'dovich and I. D. Novikov, "Gravitational Collapse of Nonsymmetric and Rotating Masses," Journal of Experimental and Theoretical Phys- 
ics (Soviet Physics JETP), Vol. 22, No. 1, 1966, pp. 122130.

http://www.jetp.ac.ru/cgi-bin/e/index/e/22/1/p122?a=list

[3] J. Winicour, A. I. Janis and E. T. Newman, "Static, Axially Symmetric Point Horizons," Physical Review, Vol. 176, No. 5, 1968, pp. 1507-1513.

http://dx.doi.org/10.1103/PhysRev.176.1507

[4] J. H. Young and C. A. Coulter, "Exact Metric for a Nonrotating Mass with a Quadrupole Moment," Physical Review, Vol. 184, No. 5, 1969, pp. 1313-1315.

http://dx.doi.org/10.1103/PhysRev.184.1313

[5] H. Quevedo, "Class of Stationary Axisymmetric Solutions of Einstein's Equations in Empty Space," Physical Review D, Vol. 33, No. 2, 1986, pp. 324-327. http://dx.doi.org/10.1103/PhysRevD.33.324

[6] H. Quevedo, "General Static Axisymmetric Solution of Einstein's Vacuum Field Equations in Prolate Spheroidal Coordinates," Physical Review D, Vol. 39, No. 10, 1989, pp. 2904-2911.

http://dx.doi.org/10.1103/PhysRevD.39.2904

[7] H. Quevedo and B. Mashhoon, "Generalization of Kerr Spacetime," Physical Review D, Vol. 43, No. 12, 1991, pp. 3902-3906.

http://dx.doi.org/10.1103/PhysRevD.43.3902

[8] J. Castejon-Amenedo and V. S. Manko, "Superposition of the Kerr Metric with the Generalized Erez-Rosen Solution," Physical Review D, Vol. 41, No. 6, 1990, pp. 20182020. http://dx.doi.org/10.1103/PhysRevD.41.2018

[9] C. Hoenselaers, W. Kinnersley and B. C. Xanthopoulos, "Symmetries of the Stationary Einstein-Maxwell Equations. VI. Transformations which generate Asymptotically Flat Spacetimes with Arbitrary Multipole Moments," Journal of Mathematical Physics, Vol. 20, No. 12, 1979, pp. 2530-2536. http://dx.doi.org/10.1063/1.524058

[10] F. J. Ernst, "New Formulation of the Axially Symmetric Gravitational Field Problem," Physical Review, Vol. 167, No. 5, 1968, pp. 1175-1177. http://dx.doi.org/10.1103/PhysRev.167.1175

[11] K. Boshkayev, H. Quevedo and R. Ruffini, "Gravitational Field of Compact Objects in General Relativity," Physical Review D, Vol. 86, No. 6, 2012, Article ID: 064043. http://dx.doi.org/10.1103/PhysRevD.86.064043

[12] A. C. Hearn, "REDUCE (User's and Contributed Packages Manual)," Konrad-Zuse-Zentrum für Informationstechnik, Berlin, 1999. http://reduce-algebra.com/

[13] J. B. Hartle and K. S. Thorne, "Slowly Rotating Relativistic Stars. II. Models for Neutron Stars and Supermassive Stars," Astrophysical Journal, Vol. 153, 1968, pp. 807834. http://dx.doi.org/10.1086/149707

[14] M. Carmeli, "Classical Fields," World Scientific Publishing, Singapore, 2001. http://www.worldscientific.com/worldscibooks/10.1142/4
$843 \# \mathrm{t}=$ toc

[15] Ya. B. Zel'dovich and I. D. Novikov, "Stars and Relativity," Dover Publications, Mineola, 2011.

[16] T. Lewis, "Some Special Solutions of the Equations of Axially Symmetric Gravitational Fields," Proceedings of the Royal Society London A, Vol. 136, No. 829, 1932, pp. 176-192.

http://rspa.royalsocietypublishing.org/content/136/829

[17] R. P. Kerr, "Gravitational Field of a Spinning Mass as an Example of Algebraically Special Metrics," Physical Review Letter, Vol. 11, No. 5, 1963, pp. 237-238. http://dx.doi.org/10.1103/PhysRevLett.11.237

[18] S. Chandrasekhar, "The Mathematical Theory of Black Holes," Oxford University Press, Oxford, 2000.

[19] S. Weinberg, "Gravitation and Cosmology: Principles and Applications of the General Theory of Relativity," John Wiley \& Sons, Inc., Hoboken, 1972.

[20] E. Berti, F. White, A. Maniopoulou and M. Bruni, "Rotating Neutron Stars: an Invariant Comparison of Approximate and Numerical Spacetime Models," Monthly Notices of the Royal Astronomical Society, Vol. 358, No. 3, 2005, pp. 923-938. http://dx.doi.org/10.1111/j.1365-2966.2005.08812.x

[21] M. A. Abramowicz, G. J. E. Almergren, W. Kluźniak and A. V. Thampan, "Circular Geodesics in the Hartle-Thorne Metric," ArXiv (gr-qc/0312070), 2003.

[22] H. Asada, "Effects of a Deformation of a Star on the Gravitational Lensing," Monthly Notices of the Royal Astronomical Society, Vol. 356, No. 4, 2005, pp. 1249-1255. http://dx.doi.org/10.1111/j.1365-2966.2004.08486.x

[23] M. H. Soffel, "Relativity in Astrometry, Celestial Mechanics and Geodesy (Astronomy and Astrophysics Library)," Springer-Verlag, Berlin, 1989. http://dx.doi.org/10.1007/978-3-642-73406-9

[24] M. H. Soffel, J. Schastok, H. Ruder and M. Schneider, "Relativistic Astrometry," Astrophysics and Space Science, Vol. 110, No. 1, 1985, pp. 95-101. http://dx.doi.org/10.1007/BF00660610

[25] J. Páez and F. Frutos, "Astrometry in the Kerr Field in PPN Approximation," Astrophysics and Space Science, Vol. 214, No. 1-2, 1994, pp. 71-87. http://dx.doi.org/10.1007/BF00982325

[26] H. Asada, M. Kasai and T. Yamamoto, "Separability of Rotational Effects on a Gravitational Lens," Physical Review D, Vol. 67, No. 4, 2003, Article ID: 043006. http://dx.doi.org/10.1103/PhysRevD.67.043006

[27] K. Yamada and H. Asada, "Post-Newtonian Effects of Planetary Gravity on the Perihelion Shift," Monthly Notices of the Royal Astronomical Society, Vol. 423, No. 4, 2012, pp. 3540-3544.

http://dx.doi.org/10.1111/j.1365-2966.2012.21150.x 


\section{Appendix}

The Ricci tensor components are

$$
\begin{aligned}
& R_{00}=\frac{1}{4 \rho^{2} Y^{2} Z^{2}}\left\{V Y Z^{2} \frac{\partial X}{\partial \theta} \frac{\partial V}{\partial \theta}+V Y^{2} Z \frac{\partial X}{\partial r} \frac{\partial V}{\partial r}+2 \rho^{2} Y Z^{2} \frac{\partial^{2} V}{\partial \theta^{2}}-X Y Z^{2}\left[\frac{\partial V}{\partial \theta}\right]^{2}-\rho^{2} Z^{2} \frac{\partial V}{\partial \theta} \frac{\partial Y}{\partial \theta}\right. \\
& \left.+\rho^{2} Y Z \frac{\partial V}{\partial \theta} \frac{\partial Z}{\partial \theta}+2 \rho^{2} Y^{2} Z \frac{\partial^{2} V}{\partial r^{2}}-X Y^{2} Z\left[\frac{\partial V}{\partial r}\right]^{2}+\rho^{2} Y Z \frac{\partial V}{\partial r} \frac{\partial Y}{\partial r}-\rho^{2} Y^{2} \frac{\partial V}{\partial r} \frac{\partial Z}{\partial r}\right\} \\
& R_{01}=R_{10}=0 \\
& R_{02}=R_{20}=0 \\
& R_{03}=R_{30}=\frac{1}{4 \rho^{2} Y^{2} Z^{2}}\left\{-2 W Y Z^{2} \frac{\partial X}{\partial \theta} \frac{\partial V}{\partial \theta}+V Y Z^{2} \frac{\partial X}{\partial \theta} \frac{\partial W}{\partial \theta}-2 W Y^{2} Z \frac{\partial X}{\partial r} \frac{\partial V}{\partial r}+V Y^{2} Z \frac{\partial X}{\partial r} \frac{\partial W}{\partial r}\right. \\
& +X Y Z^{2} \frac{\partial V}{\partial \theta} \frac{\partial W}{\partial \theta}+X Y^{2} Z \frac{\partial V}{\partial r} \frac{\partial W}{\partial r}-2 \rho^{2} Y Z^{2} \frac{\partial^{2} W}{\partial \theta^{2}}+\rho^{2} Z^{2} \frac{\partial W}{\partial \theta} \frac{\partial Y}{\partial \theta}-\rho^{2} Y Z \frac{\partial W}{\partial \theta} \frac{\partial Z}{\partial \theta} \\
& \left.-2 \rho^{2} Y^{2} Z \frac{\partial^{2} W}{\partial r^{2}}-\rho^{2} Y Z \frac{\partial W}{\partial r} \frac{\partial Y}{\partial r}+\rho^{2} Y^{2} \frac{\partial W}{\partial r} \frac{\partial Z}{\partial r}\right\} \\
& R_{11}=\frac{1}{4 \rho^{4} Y^{2} Z}\left\{-\rho^{2} V Y Z \frac{\partial X}{\partial \theta} \frac{\partial Z}{\partial \theta}-2 \rho^{2} V Y^{2} Z \frac{\partial^{2} X}{\partial r^{2}}-V^{2} Y^{2} Z\left[\frac{\partial X}{\partial r}\right]^{2}-4 \rho^{2} Y^{2} Z \frac{\partial X}{\partial r} \frac{\partial V}{\partial r}\right. \\
& +\rho^{2} V Y^{2} \frac{\partial X}{\partial r} \frac{\partial Z}{\partial r}-\rho^{2} X Y Z \frac{\partial V}{\partial \theta} \frac{\partial Z}{\partial \theta}-2 \rho^{2} X Y^{2} Z \frac{\partial^{2} V}{\partial r^{2}}-X^{2} Y^{2} Z\left[\frac{\partial V}{\partial r}\right]^{2}+\rho^{2} X Y^{2} \frac{\partial V}{\partial r} \frac{\partial Z}{\partial r} \\
& \left.+\rho^{4} Z \frac{\partial Y}{\partial \theta} \frac{\partial Z}{\partial \theta}-2 \rho^{4} Y Z \frac{\partial^{2} Y}{\partial r^{2}}+\rho^{4} Z\left[\frac{\partial Y}{\partial r}\right]^{2}+\rho^{4} Y \frac{\partial Y}{\partial r} \frac{\partial Z}{\partial r}-2 \rho^{4} Y Z \frac{\partial^{2} Z}{\partial \theta^{2}}+\rho^{4} Y\left[\frac{\partial Z}{\partial \theta}\right]^{2}\right\} \\
& R_{12}=R_{21}=\frac{1}{4 \rho^{4} Y Z}\left\{-2 \rho^{2} V Y Z \frac{\partial^{2} X}{\partial \theta \partial r}-V^{2} Y Z \frac{\partial X}{\partial \theta} \frac{\partial X}{\partial r}-2 \rho^{2} Y Z \frac{\partial X}{\partial \theta} \frac{\partial V}{\partial r}\right. \\
& +\rho^{2} V Z \frac{\partial X}{\partial \theta} \frac{\partial Y}{\partial r}-2 \rho^{2} Y Z \frac{\partial X}{\partial r} \frac{\partial V}{\partial \theta}+\rho^{2} V Y \frac{\partial X}{\partial r} \frac{\partial Z}{\partial \theta}-2 \rho^{2} X Y Z \frac{\partial^{2} V}{\partial \theta \partial r} \\
& \left.-X^{2} Y Z \frac{\partial V}{\partial \theta} \frac{\partial V}{\partial r}+\rho^{2} X Z \frac{\partial V}{\partial \theta} \frac{\partial Y}{\partial r}+\rho^{2} X Y \frac{\partial V}{\partial r} \frac{\partial Z}{\partial \theta}\right\} \\
& R_{13}=R_{31}=0 \\
& R_{22}=\frac{1}{4 \rho^{4} Y Z^{2}}\left\{-2 \rho^{2} V Y Z^{2} \frac{\partial^{2} X}{\partial \theta^{2}}-V^{2} Y Z^{2}\left[\frac{\partial X}{\partial \theta}\right]^{2}-4 \rho^{2} Y Z^{2} \frac{\partial X}{\partial \theta} \frac{\partial V}{\partial \theta}+\rho^{2} V Z^{2} \frac{\partial X}{\partial \theta} \frac{\partial Y}{\partial \theta}\right. \\
& -\rho^{2} V Y Z \frac{\partial X}{\partial r} \frac{\partial Y}{\partial r}-2 \rho^{2} X Y Z^{2} \frac{\partial^{2} V}{\partial \theta^{2}}-X^{2} Y Z^{2}\left[\frac{\partial V}{\partial \theta}\right]^{2}+\rho^{2} X Z^{2} \frac{\partial V}{\partial \theta} \frac{\partial Y}{\partial \theta} \\
& -\rho^{2} X Y Z \frac{\partial V}{\partial r} \frac{\partial Y}{\partial r}+\rho^{4} Z \frac{\partial Y}{\partial \theta} \frac{\partial Z}{\partial \theta}-2 \rho^{4} Y Z \frac{\partial^{2} Y}{\partial r^{2}}+\rho^{4} Z\left[\frac{\partial Y}{\partial r}\right]^{2}+\rho^{4} Y \frac{\partial Y}{\partial r} \frac{\partial Z}{\partial r} \\
& \left.-2 \rho^{4} Y Z \frac{\partial^{2} Z}{\partial \theta^{2}}+\rho^{4} Y\left[\frac{\partial Z}{\partial \theta}\right]^{2}\right\} \\
& R_{23}=R_{32}=0 \\
& R_{33}=\frac{1}{4 \rho^{2} Y^{2} Z^{2}}\left\{-2 \rho^{2} Y Z^{2} \frac{\partial^{2} X}{\partial \theta^{2}}+V Y Z^{2}\left[\frac{\partial X}{\partial \theta}\right]^{2}-X Y Z^{2} \frac{\partial X}{\partial \theta} \frac{\partial V}{\partial \theta}+\rho^{2} Z^{2} \frac{\partial X}{\partial \theta} \frac{\partial Y}{\partial \theta}\right. \\
& \left.-\rho^{2} Y Z \frac{\partial X}{\partial \theta} \frac{\partial Z}{\partial \theta}-2 \rho^{2} Y^{2} Z \frac{\partial^{2} X}{\partial r^{2}}+V Y^{2} Z\left[\frac{\partial X}{\partial r}\right]^{2}-X Y^{2} Z \frac{\partial X}{\partial r} \frac{\partial V}{\partial r}-\rho^{2} Y Z \frac{\partial X}{\partial r} \frac{\partial Y}{\partial r}+\rho^{2} Y^{2} \frac{\partial X}{\partial r} \frac{\partial Z}{\partial r}\right\}
\end{aligned}
$$


The scalar curvature is

$$
\begin{aligned}
R_{s}= & \frac{1}{2 \rho^{2} Y^{2} Z^{2}}\left\{2 V Y Z^{2} \frac{\partial^{2} X}{\partial \theta^{2}}+3 Y Z^{2} \frac{\partial X}{\partial \theta} \frac{\partial V}{\partial \theta}-V Z^{2} \frac{\partial X}{\partial \theta} \frac{\partial Y}{\partial \theta}+V Y Z \frac{\partial X}{\partial \theta} \frac{\partial Z}{\partial \theta}+2 V Y^{2} Z \frac{\partial^{2} X}{\partial r^{2}}\right. \\
& +3 Y^{2} Z \frac{\partial X}{\partial r} \frac{\partial V}{\partial r}+V Y Z \frac{\partial X}{\partial r} \frac{\partial Y}{\partial r}-V Y^{2} \frac{\partial X}{\partial r} \frac{\partial Z}{\partial r}+2 X Y Z^{2} \frac{\partial^{2} V}{\partial \theta^{2}}-X Z^{2} \frac{\partial V}{\partial \theta} \frac{\partial Y}{\partial \theta} \\
& +X Y Z \frac{\partial V}{\partial \theta} \frac{\partial Z}{\partial \theta}+2 X Y^{2} Z \frac{\partial^{2} V}{\partial r^{2}}+X Y Z \frac{\partial V}{\partial r} \frac{\partial Y}{\partial r}-X Y^{2} \frac{\partial V}{\partial r} \frac{\partial Z}{\partial r}-\rho^{2} Z \frac{\partial Y}{\partial \theta} \frac{\partial Z}{\partial \theta} \\
& \left.+2 \rho^{2} Y Z \frac{\partial^{2} Y}{\partial r^{2}}-\rho^{2} Z\left[\frac{\partial Y}{\partial r}\right]^{2}-\rho^{2} Y \frac{\partial Y}{\partial r} \frac{\partial Z}{\partial r}+2 \rho^{2} Y Z \frac{\partial^{2} Z}{\partial \theta^{2}}-\rho^{2} Y\left[\frac{\partial Z}{\partial \theta}\right]^{2}\right\}
\end{aligned}
$$

